\title{
Coating of polydopamine on polyurethane open cell foams to design soft structured support for molecular catalysts
}

Received 00th January 20xx, Accepted 00th January 20xx DOI: $10.1039 / \times 0 \times x 00000 x$

\author{
Ahmed Ait-Khouya, ${ }^{a}$ Miguel L. Mendez Martinez, ${ }^{b}$ Philippe Bertani, ${ }^{c}$ Thierry Romero, ${ }^{d}$ Damien \\ Favier, ${ }^{b}$ Thierry Roland, ${ }^{b}$ Valentin Guidal, ${ }^{\mathrm{e}}$ Virginie Bellière-Baca, ${ }^{\mathrm{e}}$ David Edouard, ${ }^{\mathrm{f}}$ Loïc Jierry, ${ }^{* \mathrm{~b}}$ \\ and Vincent Ritleng ${ }^{*^{\mathrm{a}, \mathrm{g}}}$
}

\begin{abstract}
Polydopamine-coated polyurethane open cell foams are used as structured support for molecular catalysts through the covalent anchoring of alkoxysilyl arms by the catechol groups of the mussel-inspired layer. This strong bonding prevents their leaching. No alteration of the mechanical properties of the flexible support is observed after repeated uses of the catalytic materials.
\end{abstract}

Continuous processes based on structured catalytic supports are advantageous at the industrial scale. Structured supports indeed display an important surface over volume ratio, and allow efficient mass transfers and small pressure drops. ${ }^{1}$ Furthermore, they generate an intimate mixing of the reagents and allow an easy separation of the catalyst from the reaction products. Among the variety of structured catalytic supports, open cell foams are prime candidates. Of ceramic or metallic constitution, these host architectures are ideal supports for catalytically active metallic nanoparticles (NPs). ${ }^{2}$ Thus, efficient solid foam bed reactors have been reported, mainly based on the coating of SiC or alumina foams with a catalytic phase. ${ }^{3}$ This kind of support have proven chemically robust for catalytic reactions under harsh conditions, showing good longevity, ${ }^{4}$ as well as enhancing the catalyst efficiency ${ }^{5}$ and selectivity ${ }^{6}$ compared to other conventional supports. Their expensive and energy consuming way of preparation however represents an important drawback for their development, especially when taking into account the current economic and ecological constraints. Moreover, these foams are heavy and difficult to handle, rigid and brittle, and present many closed cells that can alter the reproducibility of the reactions.

a. Université de Strasbourg, Ecole européenne de Chimie, Polymères et Matériaux CNRS, LIMA, UMR 7042, 25 rue Becquerel, 67087 Strasbourg, France.

${ }^{b}$. Université de Strasbourg, CNRS, Institut Charles Sadron, UPR 022, 23 rue du Loess, 67034 Strasbourg, France.

Université de Strasbourg, CNRS, Institut de Chimie, UMR 7177, 4 rue Blaise Pascal, 67081 Strasbourg, France.

d. Université de Strasbourg, Ecole européenne de Chimie, Polymères et Matériaux, CNRS, ICPEES, UMR 7515, 25 rue Becquerel, 67087 Strasbourg, France.

e. Adisseo, Antony Parc 2, 10 place Général de Gaulle, 92160 Antony, France.

f. Univ Lyon, Université Claude Bernard Lyon 1, CNRS, LAGEPP, UMR 5007, 43 boulevard du 11 novembre 1918, F-69100 Villeurbanne, France.

g. Institut Universitaire de France, 1 rue Descartes, 75231 Paris, France.

Electronic Supplementary Information (ESI) available: Experimental procedures, Figures S1-S16. See DOI: 10.1039/x0xx00000x
In this context, we have recently developed an alternative based on the use of open cell polyurethane foams (OCPUF). These inexpensive foams present similar structural and transport properties, ${ }^{2 \mathrm{~b}}$ with the advantage of being easily engineered because of their lightweight and high mechanical flexibility. ${ }^{7}$ Inspired by a biomimetic approach based on the mussels' adhesion principle, ${ }^{8}$ we have shown that the whole surface of this polymeric three-dimensional material can be efficiently coated with an adhesive layer and further functionalized with metallic or metal oxide NPs to get catalytic properties. ${ }^{9}$ The process relies on catechol chemistry and consists in coating a commercially available OCPUF with a layer of polydopamine (PDA) by simple immersion in a buffered aqueous solution of dopamine. Thanks to the adherence properties of the catechol units, ${ }^{8}$ NPs can be easily grafted all over the surface of the foam by simple dip-coating. In particular, by using $\mathrm{TiO}_{2}$ NPs, we have designed a soft structured supported photocatalyst, that can be used to degrade water pollutants under batch or flow conditions. ${ }^{9}$ Moreover, thanks to the reducing properties of the catechol units, ${ }^{10,11} \mathrm{M}(0)$ NPs can be directly generated on the surface, immobilized and stabilized, ${ }^{9 a, 11 a, b}$ which allows one to avoid the usual high temperature reduction step to form metal(0) NPs on classical supports. These properties give access to OCPUF@PDA-supported multi-site heterogeneous catalysts.

Furthermore, thanks to the presence of catechol moieties in the PDA structure, the covalent anchoring of molecular catalysts bearing a group that can react with these, such as an alkoxysilane, a chlorosilane, an amine or a thiol group should also be possible. ${ }^{8,12}$ Herein, we show that OCPUF@PDA, can indeed be functionalized with an organo- or an organometallic catalyst bearing an alkoxysilyl arm. This approach gives access to highly desirable OCPUF@PDA-supported single-site heterogeneous catalysts and opens the gate to the use of the large panel of catalysts reported for homogeneous catalysis.

According to our published procedure, ${ }^{9}$ cubic samples (4.5 $\mathrm{cm}^{3}$ ) of OCPUF (1) (20 PPI; Fig. S1- ESI) were coated with PDA by simple immersion for $16 \mathrm{~h}$ at room temperature in an aqueous solution of dopamine buffered to $\mathrm{pH} 8.5$ (Fig. 1), followed by thorough washings with deionized water. Low and 

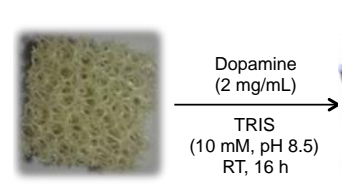

OCPUF (1)
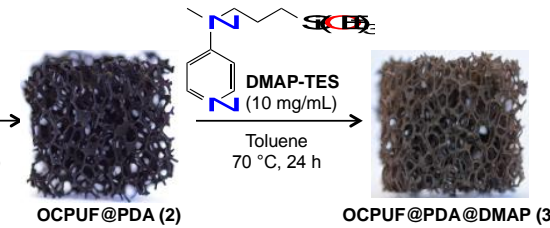

Fig. 1

OCPUF coating with PDA and covalent functionalization with DMAP-TES.

high magnification scanning electron microscopy (SEM) images of OCPUF@PDA (2) (Fig. S2) confirmed adsorption of PDA on the whole surface of the resulting black three-dimensional (3D) material as a continuous film with significant roughness, as previously observed on 3D materials ${ }^{9,11}$ and flat surfaces. ${ }^{13}$

The soft structured support $\mathbf{2}$ was then functionalized with $4-\{N$-[3-(triethoxysilyl)propyl]- $N$-methyl-amino $\}$ pyridine ${ }^{14} \quad(10$ $\mathrm{g} / \mathrm{L}$ ) by condensation of the triethoxysilyl groups with the catechol units of the PDA layer in toluene at $70^{\circ} \mathrm{C}$ for $24 \mathrm{~h}$. The black colour of $\mathbf{2}$ clears up slightly, indicating a modification of the coating (Fig. 1). The covalent anchoring of DMAP-TES to the obtained OCPUF@PDA@DMAP material 3 was confirmed by ${ }^{29} \mathrm{Si}$ CP-MAS NMR spectroscopy. The NMR spectrum indeed reveals signals at $-67,-59 \mathrm{ppm}$ and $-53 \mathrm{ppm}$ (Fig. 2A), reminiscent of the $T^{3}, T^{2}$ and $T^{1}$ substructures observed with covalently anchored DMAP-TES on a silica matrix. ${ }^{14 a}$ Inductively coupled plasma- atomic emission spectrometry (ICP-AES) measurements on several samples of $\mathbf{3}$ revealed a mean Si content of $4.44 \mathrm{~g} \pm 0.59 \mathrm{~g} / \mathrm{kg}$ (i.e. $0.158 \pm 0.021$ $\mathrm{mol} / \mathrm{kg}$ ). Low magnification SEM images reveal a slight deformation of the struts whose edges become corrugated, as well as the rough surface characteristic of the PDA coating. Higher magnification images show the presence of micrometric nodules randomly dispersed all over the surface of the functionalized material $\mathbf{3}$ (Fig. 3 and S3), suggesting a possible accumulation of DMAP in the latter. Elemental mapping of Si by SEM-energy dispersive X-ray spectroscopy (EDX) however revealed a relatively homogeneous distribution of this element on the surface of $\mathbf{3}$ (Fig. S4 and S5), and thus of
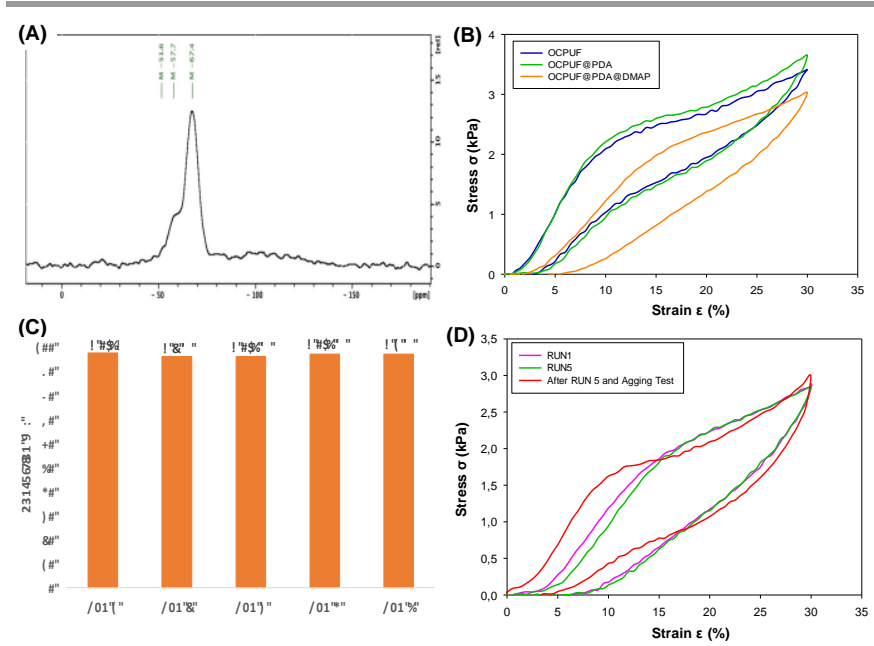

Fig. 2 (A) ${ }^{29}$ Si CP-MAS NMR spectrum of 3 (B) Stress/strain responses of 1 (violet), (green), as-synthesized $\mathbf{3}$ (orange). (C) Acylation (\%) of benzyl alcohol catalyzed by $\mathbf{3}$ after $24 \mathrm{~h}$ reaction for runs 1 to 5 ; average values obtained with 3 different foams. (D) Stress/strain responses of one foam $\mathbf{3}$ used for the recycling tests after run 1 (pink), run 5 (green), and after the aging test (red).

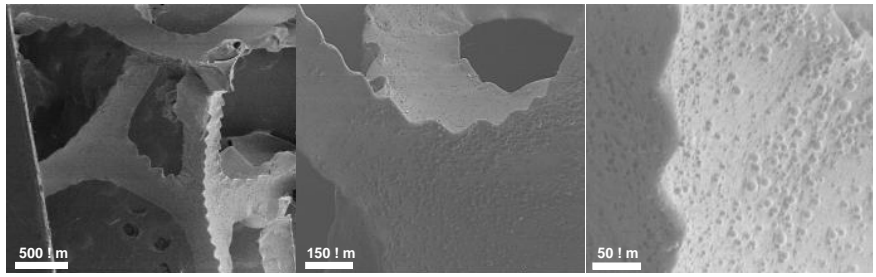

Fig. 3 SEM images of OCPUF@PDA@DMAP (3) with different magnifications

DMAP onto 2, rather than a strengthen Si signal in these nodules. Hence, we expect that, the solvent, i.e. toluene, is diffusing within the polyurethane material during the silanization step, leading to a slight swelling of the foam, and that the removal of the solvent under vacuum generates this kind of "bubbles" on the surface of the foam. Accordingly a decrease of about $3 \%$ of the foam mass was observed.

Compression tests (up to $30 \%$ deformation) were then performed on pristine $\mathbf{1}, \mathbf{2}$, and $\mathbf{3}$ to evaluate their chemical resistance through their respective stress/strain responses (Fig. 2B and S6). These measurements show typical hysteresis loop for all three foams. The resulting Young modulus values of $29.7 \mathrm{kPa}$ for $\mathbf{1}$ and $33.3 \mathrm{kPa}$ for $\mathbf{2}$ (Fig. S7) indicate that the PDA deposition do not significantly modify the mechanical properties of $\mathbf{2}$ compared to 1 . $^{9}$ These Young modulus values also reveal a slightly lower stress response to the enforced strain for $\mathbf{3}$ (18.5 $\mathrm{kPa}$ ) compared to $\mathbf{1}$ and $\mathbf{2}$. As low-density open cell foams mainly deform by bending of their cell edges, a quadratic dependence on the relative density is usually observed for the elastic modulus. ${ }^{15}$ Accordingly, calculation demonstrated that the small reduction of the Young's modulus observed for $\mathbf{3}$ is due both to a lowering of the foam density by about 3\%, as measured experimentally, and to the induced corrugated shape of the cell edges (Fig. S8 and S9).

The catalytic activity of $\mathbf{3}$ was next investigated for the acylation of alcohols as a model reaction. ${ }^{16}$ Initial studies focused on the acylation of benzyl alcohol (0.25 M) with 1.4 equivalent of acetic anhydride at $30{ }^{\circ} \mathrm{C}$ in the presence of 0.6 mol\% of DMAP, DMAP-TES or DMAP moiety anchored onto OCPUF@PDA in various solvents (Table 1). With DMAP, full conversion to the acylated alcohol was observed after $3 \mathrm{~h}$ reaction in $n$-hexane (entry 1 ). With DMAP-TES, only $84 \%$ conversion was observed after $3 \mathrm{~h}$ in $n$-hexane (entry 2 ). Using a 1:1 mixture of acetone and $n$-hexane instead, allowed observing full conversion in $3 \mathrm{~h}$ with DMAP-TES as well (entry $3)$. This solvent was thus chosen for the rest of the study. Satisfyingly, 3 allowed $81 \%$ conversion after $3 \mathrm{~h}$ (TOF $=45 \mathrm{~h}^{-1}$ vs. $56 \mathrm{~h}^{-1}$ for DMAP in $n$-hexane; entries 1 and 4 ) and $97 \%$ after $19 \mathrm{~h}$ (entry 5). Control experiments with 1 and $\mathbf{2}$ showed no conversion in both cases (entry 6), thus demonstrating that it is well the immobilized DMAP moiety that catalyses the acylation reaction. Furthermore, ICP-AES and HRMS analyses of the reaction medium at the end of the acylation with 3 revealed no traces of silicon and no fragments of DMAP-TES, which shows the absence of catalyst leaching. This was further demonstrated by a stop-and-go experiment that showed that the catalysis stops when $\mathbf{3}$ is removed from the reaction medium, and re-starts when it is re-immersed (Fig. S10). 


\begin{tabular}{|c|c|c|c|c|c|}
\hline & $=0.25 \mathrm{M}$ & 4 equiv. & & & \\
\hline Entry & Catalyst & Solvent & Time (h) & Conv. $(\%)^{b}$ & $\operatorname{TOF}\left(\mathrm{h}^{-1}\right)^{c}$ \\
\hline 1 & DMAP & $n$-hexane & 3 & 100 & 56 \\
\hline 2 & DMAP-TES & $n$-hexane & 3 & 84 & 47 \\
\hline 3 & DMAP-TES & $n$-hexane/acetone & 3 & 100 & 56 \\
\hline $4^{d, e}$ & 3 & $n$-hexane/acetone & 3 & 81 & 45 \\
\hline $5^{d, e}$ & 3 & $n$-hexane/acetone & 19 & 97 & 9 \\
\hline $6^{e, f}$ & 1 or 2 & $n$-hexane/acetone & 3 & 0 & 0 \\
\hline
\end{tabular}

${ }^{a}$ Reaction conditions: benzyl alcohol $(6.3 \mathrm{mmol}), \mathrm{Ac}_{2} \mathrm{O}(8.7 \mathrm{mmol})$, catalyst $(0.6$ mol\%) in the solvent $(25 \mathrm{~mL})$ at $30{ }^{\circ} \mathrm{C} .{ }^{b}$ Conversions determined by ${ }^{1} \mathrm{H} \mathrm{NMR} .{ }^{c} \mathrm{TOF}$ values calculated from the conversions observed at the end of the reaction times ${ }^{d}$ A cubic piece of $\mathbf{3}$, whose mass was adjusted to have $0.6 \mathrm{~mol} \%$ of immobilized DMAP-TES based on the Si content (i.e. $239 \mathrm{mg}$ of 3 for $4.44 \mathrm{~g} \mathrm{Si} / \mathrm{kg}$ ), was immersed in the solution that was stirred at a rate of $600 \mathrm{rpm} .{ }^{e} n$ Hexane/acetone ratio of $1: 1 .{ }^{f}$ A cubic piece of $1(224 \mathrm{mg})$ or 2 (226 mg) was immersed in the solution that was stirred at a rate of $600 \mathrm{rpm}$.

The scope of the acylation reaction was then briefly investigated with 3 in $n$-hexane/acetone $(1: 1)(25 \mathrm{~mL})$ at $30{ }^{\circ} \mathrm{C}$ for $24 \mathrm{~h}$ (Table 2). $98 \%$ conversion to benzylacetate was obtained under these conditions (entry 1 ). Both electron-rich and electron-poor benzyl alcohols reacted well, giving excellent conversions (entries 2 and 3). $p$-Aminobenzyl alcohol was fully converted but provided a 7:3 mixture of $p$ aminobenzyl acetate and $p$-(acylamino)benzyl acetate (entry 4). Phenol, furfuryl, allyl and cynnamyl alcoholswere all converted to the corresponding acetates with good to excellent yields (entries 5 to 8). The secondary alcohol, cyclohexanol, gave only $50 \%$ conversion (TOF $=3.5 \mathrm{~h}^{-1}$ ) (entry 9). This last result nevertheless compare advantageously with that obtained with DMAP-TES functionalized mesoporous silica nanospheres that required a catalytic charge of $7.5 \mathrm{~mol} \%$, a temperature of $60{ }^{\circ} \mathrm{C}$, as well as the presence of $\mathrm{NEt}_{3}$ to achieve this acylation with a similar TOF of $4.8 \mathrm{~h}^{-1}$. ${ }^{14 a}$

The reusability and chemical resistance of $\mathbf{3}$ was next examined by carrying out five consecutive runs of benzyl alcohol acylation under the conditions of Table 2. After run 1 and 5, 3 was removed from the reaction medium, washed with $n$-hexane/acetone (1:1), dried under vacuum and submitted to a stress/strain test. The goal of this analysis was to determine the impact of successive immersions of $\mathbf{3}$ in an organic medium at $30{ }^{\circ} \mathrm{C}$ on the mechanical properties of the polymeric support. As shown in Figure $2 \mathrm{C}$, the catalytic activity remained remarkably constant during the five consecutive runs. This was checked with three different foams. Furthermore, the stress/strain response recorded after run 5 was similar to that recorded before the five runs and after run 1 (Fig. 2D), which shows that the mechanical properties of the flexible catalytic material are not affected by its repeated use in $n$-hexane and acetone, and in the presence of a corrosive reagent such as acetic anhydride. Finally, to evaluate the mechanical resistance of $\mathbf{3}$ over time, a fatigue test consisting in compressing 35000 times to a strain of $30 \%(1 \mathrm{~Hz})$ after run 5 was carried out (Fig. 2D). An almost unchanged Young
Table 2 Acylation of alcohols catalyzed by COPUF@PD\#@DMAP (3) ${ }^{a, b}$

\begin{tabular}{|c|c|c|c|}
\hline Entry & Substrate & $\mathrm{R}$ & Conv. $(\%)^{c}$ \\
\hline 1 & & $\mathrm{R}=\mathrm{H}$ & 98 \\
\hline 2 & & $\mathrm{R}=\mathrm{OMe}$ & 90 \\
\hline 3 & & $R=F$ & 93 \\
\hline 4 & & $\mathrm{R}=\mathrm{NH}_{2}$ & $99^{d}$ \\
\hline 5 & & - & 95 \\
\hline & & - & 96 \\
\hline 7 & & - & 86 \\
\hline 8 & & - & 96 \\
\hline 9 & & - & 50 \\
\hline
\end{tabular}

${ }^{a}$ Reaction conditions: alcohol $(6.3 \mathrm{mmol}), \mathrm{Ac}_{2} \mathrm{O}(8.7 \mathrm{mmol})$, catalyst $(0.6 \mathrm{~mol} \%)$ in $n$-hexane/acetone $(1: 1)(25 \mathrm{~mL})$ at $30{ }^{\circ} \mathrm{C}$ for $24 \mathrm{~h} .{ }^{b}$ A single piece of 3 , whose mass was adjusted to have $0.6 \mathrm{~mol} \%$ of immobilized DMAP-TES based on the Si content (i.e. $239 \mathrm{mg}$ of $\mathbf{3}$ for $4.44 \mathrm{~g} \mathrm{Si} / \mathrm{kg}$ ), was immersed in the solution that was stirred at a rate of $600 \mathrm{rpm} .{ }^{C}$ Conversions determined by ${ }^{1} \mathrm{H}$ NMR; average values of two runs. ${ }^{d}$ A 7:3 mixture of $p$-aminobenzyl acetate and $p$-(acylamino)benzyl acetate was formed.

modulus $(20.7 \mathrm{kPa})$ is observed for the artificially aged 3 compared to as-synthesized $3(18.5 \mathrm{kPa}), 3$ after run 1 (18.7 $\mathrm{kPa}$ ) and after run 5 (17.5 $\mathrm{kPa}$ ) (Fig. S7), showing that $\mathbf{3}$, overall, keeps its elastic properties overtime; a feature that could allow to modulate the shape of the internal architecture of the support through the application of an external mechanical force that could reversibly deform the catalytic bed in order to modulate/optimize the morphological parameters of the foam and thus its transport properties. In contrast to rigid open cell foams with fixed morphological parameters and transport properties, using soft structured foam could thus allow to tune these properties according to the catalytic reaction needs. Furthermore, increased mass transfer should be expected during compression/stretching cycles. ${ }^{17}$ These considerations represent, we believe, a change of paradigm in chemical engineering.

Encouraged by these results, we then decided to test further the longevity of $\mathbf{3}$ by conducting 10 consecutive runs of benzyl alcohol acylation of 3 to $19 \mathrm{~h}$ and then checking the consequences of these runs on the PDA@DMAP layer by SEM. Again, the activity was found to remain remarkably constant overtime (Fig. S11). In agreement with this longevity, only minor changes were observed at the surface. Thus, low magnification SEM images of the so used $\mathbf{3}$ still show corrugated edges, and higher magnification images still show micrometric nodules, even though most of them have vanished and a rough surface with dispersed nanometric aggregates is now seen, as typically observed with PDA coatings (Fig. S12)..$^{9,11,13}$

Finally, to establish the versatility of our approach to design a soft structured support for molecular catalysts, we next investigated the covalent anchoring of an organometallic complex. For that purpose, we used the nickel-NHC complex 4 (Fig. 4 and Fig. S13-S17), whose analogues have been shown 


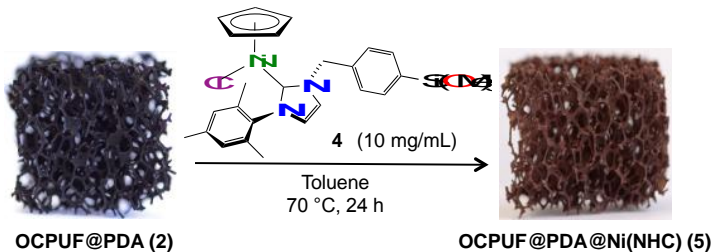

Fig. 4

Covalent functionalization of OCPUF@PDA (2) with 4.

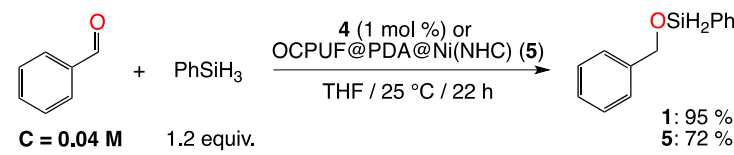

Scheme 1. Hydrosilylation of benzaldehyde catalyzed by $\mathbf{4}$ and 5 .

to efficiently catalyse the hydrosilylation of carbonyl derivatives. ${ }^{18}$ Similarly than with DMAP-TES, 4 was grafted on 2 by condensation of its alkoxysilyl groups with the catechol units of the PDA layer in toluene at $70^{\circ} \mathrm{C}$ for $24 \mathrm{~h}$ (Fig. 4).

After characterization by SEM (Fig. S18), and assessment of the nickel content by ICP-AES analyses of several samples of OCPUF@PDA@Ni(NHC) (5) (4.24 g \pm 0.49 g/kg, i.e.: $72.2 \pm 8.3$ $\mathrm{mmol} / \mathrm{kg}$ ), we carried out a brief catalytic study to establish the possibility to also graft organometallic catalysts onto $\mathbf{2}$. For that purpose, we investigated the catalytic activity of $\mathbf{5}$ (mass adjusted to have 1 mol\% of immobilized complex based on the $\mathrm{Ni}$ content) for the hydrosilylation of benzaldehyde $(0.04 \mathrm{M})$ in THF at $25{ }^{\circ} \mathrm{C}$ with $\mathrm{PhSiH}_{3}$ (1.2 equiv.) as the hydrogen source, ${ }^{17 \mathrm{~b}}$ and compared it to that of 4 (1 mol\%) under similar conditions (Scheme 1). After $22 \mathrm{~h}, 95 \%$ conversion to the silyl ether was observed with $4\left(\right.$ TOF $=4.3 \mathrm{~h}^{-1}$ ) and $72 \%$ with 5 (TOF $=3.3 \mathrm{~h}^{-1}$ ). This demonstrates the possibility to immobilize both organo- and organometallic catalysts on OCPUF@PDA (2) and thus to potentially access to a multitude of catalysts.

In summary, polydopamine has been used as an adhesive layer on soft structured supports made of flexible polyurethane open cell foams to covalently immobilize organoand organometallic catalysts through a silanization process. This convergent grafting strategy, never reported onto PDA, allows anchoring organo- and organometallic catalysts as long as they are modified with suitable silane groups. This paves the way to the access of a large panel of possible chemical transformations. Furthermore, the ability of the musselinspired coating to both bind tightly to the foam and form strong covalent bonds with the molecular catalysts avoids catalyst leaching into the medium. This property, combined to the high chemical resistance of both the polyurethane foam and the polydopamine layer, leads to easy-to-handle longliving catalysts, as shown by the repeated uses of $\mathbf{3}$ and the conservation of its mechanical properties. Thanks to its lightweight, flexibility and low-pressure drop, such kind of soft structured catalytic support thus appears as an ideal candidate to design original flow chemistry ${ }^{2,4,19}$ or dynamic bed reactors. ${ }^{17}$ This pioneering work, together with our previous work that showed the possibility to also immobilize NPs, ${ }^{9}$ opens the way to a huge array of uses of $\mathbf{2}$ as a macroscopic soft structured support for chemical engineering development.

\section{Conflicts of interest}

There are no conflicts to declare.

\section{Notes and references}

1 J. J. W. Bakker, W. J. Groendijk, K. M. de Lathouder, F. Kapteijn, J. A. Moulijn, M. T. Kreutzer and S. A. Wallin, Ind. Eng. Chem. Res., 2007, 46, 8574.

2 (a) L. Giani, G. Groppi and E. Tronconi, Ind. Eng. Chem. Res., 2005, 44, 4993; (b) J. T. Richardson, Y. Peng and D. Remue, Appl. Catal. A, 2000, 204, 19; (c) M. Lacroix, P. Nguyen, D. Schweich, C. Pham Huu, S. Savin-Poncet and D. Edouard, Chem. Eng. Sci., 2007, 62, 3259.

3 C. Duong-Viet, H. Ba, Z. El-Berrichi, J.-M. Nhut, M. J. Ledoux, Y. Liu and C. Pham-Huu, New. J. Chem., 2016, 40, 4285.

4 X. Ou, F. Pilitsis, Z. Jiao, Y. Zhang, S. Xu, M. Jennings, Y. Yang, S. F. Rebecca Taylor, A. Garforth, H. Zhang, C. Hardacre, Y. Yan and X. Fan, Chem. Eng. J., 2019, 362, 53.

5 L. Feng, Y. Liu, Q. Jiang, W. Liu, K.-H. Wu, H. Ba, C. Pham-Huu, W. Yang and D. Sheng Su, Catal. Today, 2019, doi.org/10.1016/j.cattod.2019.02.046.

6 X. Jiao, X. Ou, J. Zhang and X. Fan, React. Chem. Eng., 2019, $4,427$.

7 H.-W. Engels, H.-G. Pirkl, R. Albers, R. W. Albach, J. Krause, A. Hoffmann, H. Casselmann and J. Dormish, Angew. Chem. Int. Ed., 2013, 52, 9422.

8 H. Lee, S. M. Dellatore, W. M. Miller and P. M. Messersmith, Science, 2007, 318, 426.

9 (a) D. Edouard, V. Ritleng, L. Jierry and N. T. T. ChauDalencon, WO 2016012689A2, 2016; (b) E. Pardieu N. T. T. Chau, T. Dintzer, T. Romero, D. Favier, T. Roland, D. Edouard, L. Jierry and V. Ritleng, Chem. Commun., 2016, 52, 4691.

10 V. Ball, I. Nguyen, M. Haupt, C. Arnoult, V. Toniazzo and D. Ruch, J. Coll. Interf. Sci., 2011, 364, 359.

11 (a) L. Lefebvre, J. Kelber, L. Jierry, V. Ritleng and D. Edouard, J. Environ. Chem. Eng., 2017, 5, 79; (b) D. Edouard, L. Lefebvre, L. Jierry, V. Ritleng and J. Kelber, WO 2018020146 A1, 2018; (c) L. Lefebvre, J. Kelber, X. Mao, F. Ponzio, G. Agusti, C. Vigier-Carrière, V. Ball, L. Jierry, V. Ritleng and D. Edouard, Environ. Prog. Sustain. Energy, 2019, 38, 329.

12 O. Pop-Georgievski, D. Verreault, M.-O. Diesner, V. Proks, S. Heissler, F. Rypáček and P. Koelsch, Langmuir, 2012, 28, 14273.

13 R. A. Zangmeister, T. A. Morris, and M. J. Tarlov, Langmuir, 2013, 29, 8619.

14 (a) H.-T. Chen, S. Huh, J. W. Wiench, M. Pruski and V. S.-Y. Lin, J. Am. Chem. Soc., 2005, 127, 13305-13311; (b) N. A. Brunelli, W. Long, K. Venkatasubbaiah and C. W. Jones, Top. Catal., 2012, 55, 432.

15 W. E. Warren and A. M. Kryanik, J. Appl. Mech., 1997, 64, 787.

16 (a) G. Höfle, W. Steglich and H. Vorbriiggen, Angew. Chem., Int. Ed. Engl., 1978, 17, 569; (b) A. C. Spivey and S. Arseniyadis, Angew. Chem. Int. Ed., 2004, 43, 5436.

17 Z.-C. Hu, R. A. Korus and K. E. Stormo, Appl. Microbio. Biotech., 1993, 39, 289.

18 (a) L. P. Bheeter, M. Henrion, L. Brelot, C. Darcel, M. J. Chetcuti, J.-B. Sortais and V. Ritleng, Adv. Synth. Catal., 2012, 354, 2619; (b) F. Ulm, A. I. Poblador-Bahamonde, S. Choppin, S. Bellemin-Laponnaz, M. J. Chetcuti, T. Achard and V. Ritleng, Dalton Trans., 2018, 47, 17134.

19 (a) D. Edouard, M. Lacroix, C. Pham, M. Mbodji and C. Pham-Huu, AIChE J., 2008, 53, 2823; (b) S. Josset, S. Hajiesmailia, D. Begin, D. Edouard, C. Pham-Huu, M.-C. Lett, N. Keller and V. Keller, J. Hazard. Mater., 2010, 175, 372. 